\title{
Retrosigmoid Craniotomy for the Removal of Left Sided Tentorial and Posterior Fossa Meningioma Combined with Microvascular Decompression for Hemifacial Spasm
}

\author{
Yu-Wen Cheng ${ }^{1,2,3,5}$ Chun-Yu Cheng ${ }^{3,4,5}$ Zeeshan Qazi ${ }^{3,5} \quad$ Laligam N. Sekhar ${ }^{3,5}$ \\ ${ }^{1}$ Graduate Institute of Clinical Medicine, Kaohsiung Medical \\ Address for correspondence Yu-Wen Cheng, MD, Neurosurgical \\ University, Taiwan \\ Department, Kaohsiung Veterans General Hospital, No.386, Dazhong \\ 2 Department of Neurosurgery, Kaohsiung Veterans General Hospital, \\ Taiwan \\ ${ }^{3}$ Department of Neurosurgery, University of Washington, Seattle, \\ First Road, Zuoying District, Kaohsiung 81362, Taiwan \\ Washington, United States \\ ${ }^{4}$ Department of Neurosurgery, Chang Gung University Hospital, \\ Chiayi; College of Medicine, Chang Gung University, Taiwan \\ ${ }^{5}$ Department of Neurosurgery, Harborview Medical Centre, Seattle, \\ Washington, United States \\ J Neurol Surg B 2019;80(suppl S3):S294-S295.
}

\begin{abstract}
This 68-year-old woman presented with repeated episodes of bilateral hemifacial spasm with headache for 5 years and with recent progression of left sided symptoms. Preoperative imaging showed a left sided tentorial meningioma with brain stem and cerebellar compression. Left facial nerve was compressed by the vertebral artery (VA) and the right facial nerve by the anterior inferior cerebellar artery (AICA). This patient underwent left side retrosigmoid craniotomy and mastoidectomy. The cisterna magna was drained to relax the brain. The tumor was very firm, attached to the tentorium and had medial and lateral lobules. The superior cerebellar artery was adherent to the lateral lobule of the tumor and dissected away. The tumor was detached from its tentorial base; we first removed the lateral lobule. Following this, the medial lobule was also completely dissected and removed. The root exit zone of cranial nerve (CN) VII was dissected and exposed. The compression was caused both by a prominent VA and AICA. Initially, the several pieces of Teflon felt were placed for the decompression. Then vertebropexy was performed by using 8-0 nylon

\section{Keywords}

- tentorial meningioma

- microvascular decompression

- hemifacial spasm

- vertebropexy suture placed through the VA media to the clival dura. A further piece of Teflon felt was placed between cerebellopontine angle region and AICA. Her hemifacial spasm resolved postoperatively, and she discharged home 1 week later. Postoperative imaging showed complete tumor removal and decompression of left CN VII. This video shows the complex surgery of microsurgical resection of a large tentorial meningioma and microvascular decompression with a vertebropexy procedure.

The link to the video can be found at: https://youtu.be/N5aHN9CRJeM.
\end{abstract}

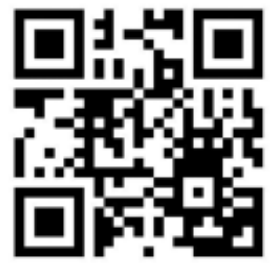

received

May 23, 2018

accepted

August 19, 2018

published online

October 18, 2018 www.thieme.com/skullbasevideos

www.thieme.com/jnlsbvideos
License terms

10.1055/s-0038-1675167. ISSN 2193-6331. (c) 2019 Georg Thieme Verlag KG Stuttgart · New York

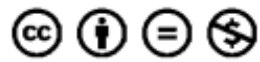




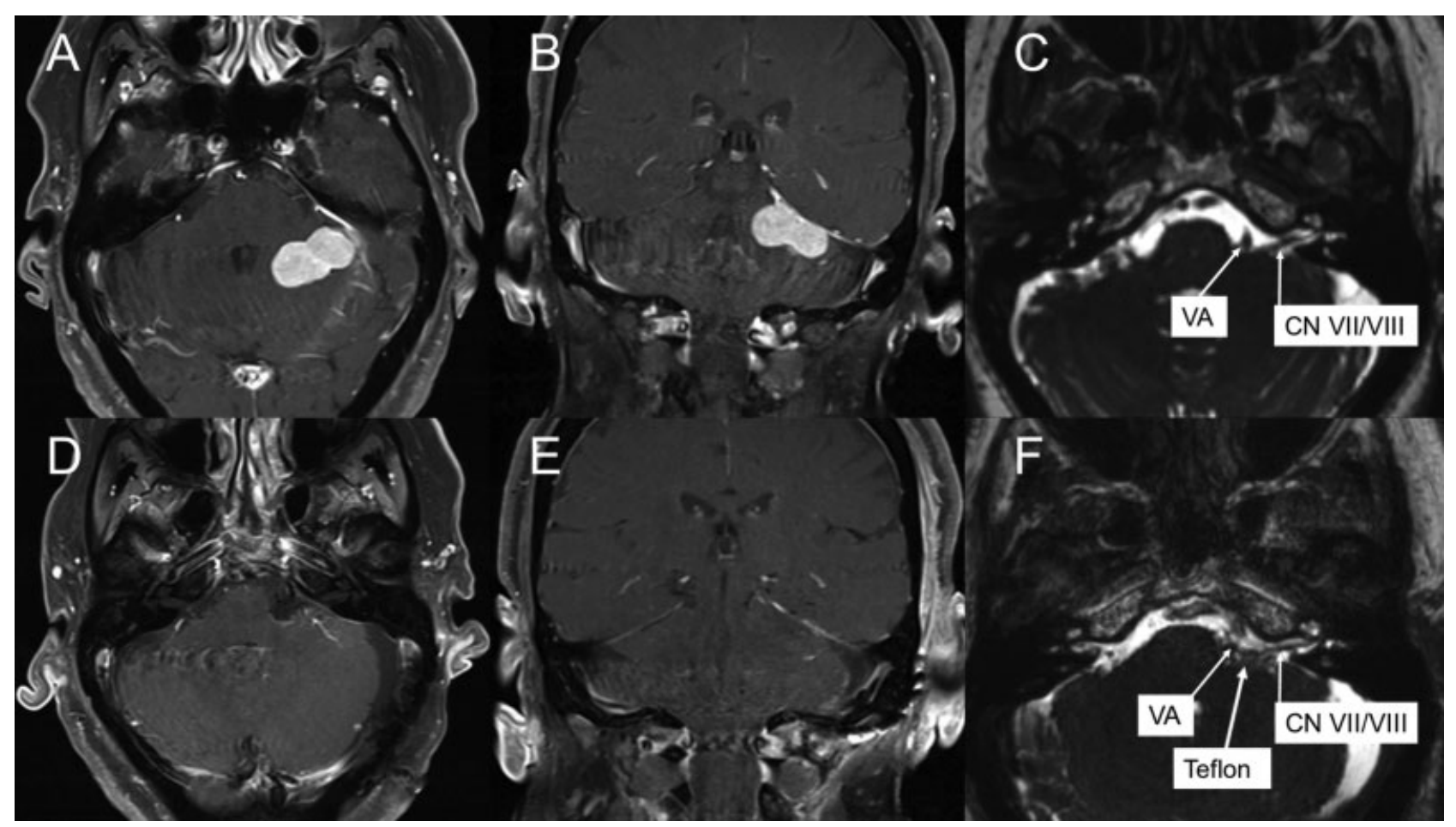

Fig. 1 Preoperative Brain MRI with gadolinium contrast (A) axial and (B) coronal view showing a left sided bilobule tentorial meningioma with brain stem and cerebellar compression; (C) showed left facial nerve was compressed by the VA. At 3 days follow-up, postoperative (D) axial and (E) coronal brain MRI with gadolinium contrast demonstrating complete resection of the lesion and showed VA was separated from facial nerve by (F) Teflon felt. MRI, magnetic resonance imaging; VA, vertebral artery.

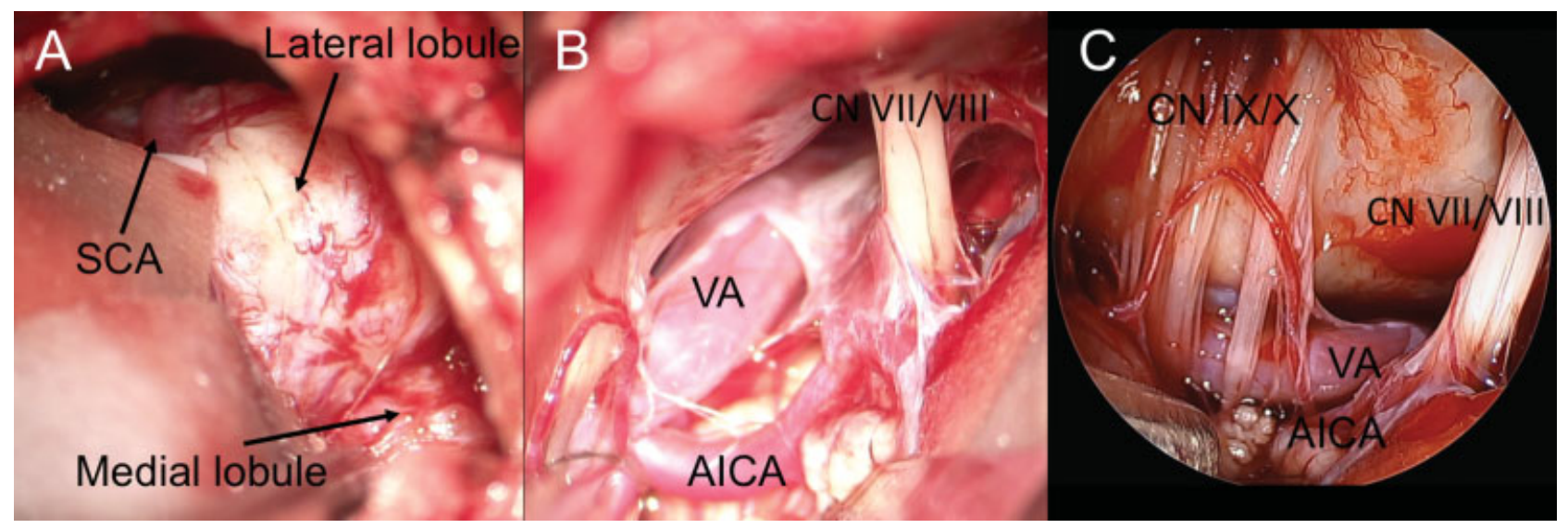

Fig. 2 Intraoperative image revealed (A) tumor was firmly attached to the tentorium and had medial and lateral lobules; the superior cerebellar artery was adherent to the lateral lobule. The root exit zone of CN VII was dissected and exposed. The compression was caused both by a prominent VA and AICA from the microscopic view (B) and endoscopic view (C). AICA, anterior inferior cerebellar artery; CN, cranial nerve; SCA, superior cerebellar artery; VA, vertebral artery.

Conflict of Interest

None. 